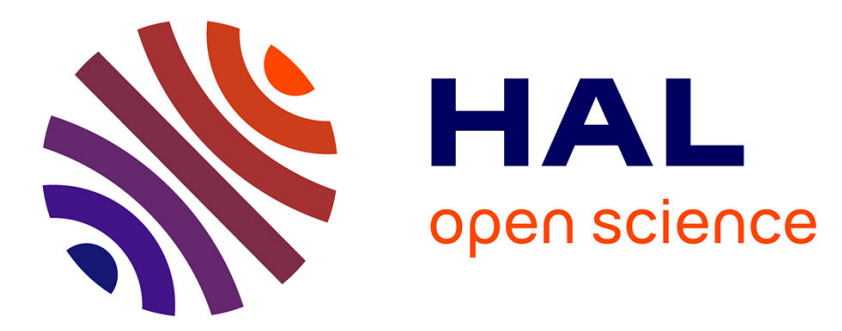

\title{
INVARIANT REPRESENTATION OF PROPAGATION PROPERTIES FOR BI-COUPLED PERIODIC STRUCTURES
}

Francesco Romeo, Angelo Luongo

\section{- To cite this version:}

Francesco Romeo, Angelo Luongo. INVARIANT REPRESENTATION OF PROPAGATION PROPERTIES FOR BI-COUPLED PERIODIC STRUCTURES. Journal of Sound and Vibration, 2002, 257 (5), pp.869-886. hal-00798016

\section{HAL Id: hal-00798016 https://hal.science/hal-00798016}

Submitted on 7 Mar 2013

HAL is a multi-disciplinary open access archive for the deposit and dissemination of scientific research documents, whether they are published or not. The documents may come from teaching and research institutions in France or abroad, or from public or private research centers.
L'archive ouverte pluridisciplinaire HAL, est destinée au dépôt et à la diffusion de documents scientifiques de niveau recherche, publiés ou non, émanant des établissements d'enseignement et de recherche français ou étrangers, des laboratoires publics ou privés. 


\title{
INVARIANT REPRESENTATION OF PROPAGATION PROPERTIES FOR BI-COUPLED PERIODIC STRUCTURES
}

\author{
F. Romeo And A. LUONGo \\ Dipartimento di Ingegneria delle Strutture, delle Acque e del Terreno, Università di L'Aquila, 67040 \\ Monteluco di Roio, L'Aquila, Italy.E mail: romeo@ing.univaq.it
}

\begin{abstract}
General bi coupled periodic systems are dealt with by means of transfer matrices of single units. The solutions of the associated characteristic equation are discussed in terms of invariant quantities by exploiting the well known reversibility of its coefficients. An exhaustive description of the free wave propagation patterns is given on the invariant plane where propagation domains with qualitatively different character are identified. The asymptotic behavior of the roots of the characteristic equation when the invariants tend to infinity is analyzed. The contour plot of the real part of the propagation constants, responsible for the amount of attenuation of the characteristic waves, is illustrated on the invariants' plane. Next, several models of bi coupled periodic structures made up of beams resting on elastic supports are considered. A non linear mapping from the invariants' plane to the physical parameters plane provides a concise representation of the pattern of the propagation domains. A mechanical interpretation associated with the boundaries of these regions is given. Finally, the proper selection of the physical parameters governing the propagation modes is discussed.
\end{abstract}

\section{INTRODUCTION}

Periodic structures consist of a series of identical elements joined together. The transfer matrix method has been extensively adopted in literature to analyze the dynamics of such structures [1, 2]. This method features the computational advantage of reducing the dimension of the underlying problem to the number of degrees of freedom (d.o.f.) coupling basic periodic elements. Such dimension is thereby independent of the number of elements constituting the whole structure. The dynamic behavior of mono-coupled systems (1 d.o.f. at the interface) has been thoroughly addressed [3]; already in the late 1960 s it was evidenced that the disturbance propagation through mono-coupled periodic structures is governed by the frequency-dependent transfer matrix eigenvalues. On the frequency axis there exist intervals or bands where disturbances propagate harmonically without attenuation (pass-bands), in which the eigenvalues are complex with unit modulus, and bands where the disturbances decay (stop-bands), in which the eigenvalues are real and different from 1. Analytical studies have also been proposed for bi-coupled periodic structures [4] while mostly numerical approaches have been developed for multi-coupled cases [5, 6]. It has been found that as soon as the coupling co-ordinates are more than one, there exist further frequency bands characterized by disturbance harmonic propagation with attenuation (complex-bands) where pairs of complex conjugate eigenvalues, with modulus different from 1, exist. 
The description of the dynamics of multi-coupled periodic structures so far proposed in literature has relied on the frequency as the sole parameter [7-11]. Nevertheless, such description is generally not exhaustive of the propagation properties of the system, since it qualitatively depends on the values fixed for the remaining physical parameters. Indeed, when these are modified, some existing bands generally disappear and some new bands appear somewhere. Therefore, a multi-dimensional representation is necessary to completely depict the propagation properties scenario. However, not all the parameters have the same qualitative influence on such properties, so that the problem of selecting the smallest set of parameters, able to furnish a complete representation, arises. The task has some similarities with that of linear bifurcation analysis of dynamical systems [12]. The most natural way to solve the problem is to refer to the invariants $I_{k}$ of the transfer matrix characteristic equation that, for a $2 n$-coupled system reads: $\lambda^{2 n}+\sum_{k=1}^{2 n} I_{k} \lambda^{2 n k}=0$, where $I_{k} \in \mathbb{R}$, if the system is undamped. Thanks to the coefficients reversibility $I_{k}=I_{2 n k}$ (which entails that $\lambda_{h}$ and $\lambda_{h}{ }^{1}$ are both roots of the polynomial), the eigenvalues $\lambda_{h}$ only depend on $n$ real quantities $I_{j}(j=1, \ldots, n)$, whatever the number of the physical parameters is. Therefore, by identifying in the $n$-dimensional space $\left\{I_{j}\right\}$ the domains in which the $2 n$ eigenvalues $\lambda_{h}$ are of the same type, an exhaustive geometrical representation of the propagation properties is achieved.

Such description is universal, as it is system-independent. However, when dealing with a specific $n$-coupled system, it would be desirable to transform the invariant space into a physical space. This transformation is accomplished by expressing the invariants $I_{j}$ as functions of $n$ chosen parameters $\mathbf{p}_{c}$, which will be referred to as control parameters, namely $I_{j}=I_{j}\left(\mathbf{p}_{c}\right)$. These relationships map the invariant into the physical space. However, since the relations are in general of a non-linear type, one point of the former space is mapped in more points of the latter space. Thus the connected domains of the invariant representation split into disjoined domains in the physical parameter plane, thus explaining the bands alternation in the usual one-dimensional representation. Among the control parameters, the frequency is the most significant one and should be included in the set as a distinguished control parameter. The choice of the remaining $(n-1)$ control parameters is arbitrary, whilst it is expected that they are not all equivalent. The optimum choice would consist in obtaining a representation qualitatively independent of the remaining (auxiliary) parameters, at least in some range of their values. No general criteria are so far available, but only examples are shown here to address the problem.

In this work a qualitative analysis carried out on the invariants' plane of bi-coupled periodic structures is proposed. It enables both to identify the stop, pass and complex domains and to analytically derive the boundaries of such regions. Symmetric and unsymmetric structures made up of beams resting on elastic supports with both distributed and lumped masses are considered and their behavior described on the physical parameters space. The role played by the physical parameters in the wave propagation patterns is eventually discussed.

\section{INVARIANT PROPAGATION SCENARIOS FOR BI COUPLED PERIODIC STRUCTURES}

A generic periodic structure whose elements are coupled through $n=2$ d.o.f. to the adjacent ones is considered. The dynamic behavior of such bi-coupled structures is conveniently described by means of the transfer matrix method. Let $\mathbf{z}_{k}=\left(\mathbf{q}_{k}, \mathbf{f}_{k}\right)^{\mathrm{T}}$ be the state vector of generalized displacement $\mathbf{q}_{k}$ and forces $\mathbf{f}_{k}$ at the coupling point $k$; according to the transfer matrix approach, the state vector $\mathbf{z}_{k+1}$ at the coupling point $(k+1)$ is 
related to the state vector $\mathbf{z}_{k}$ by

$$
\mathbf{z}_{k+1}=\mathbf{T} \mathbf{z}_{k},
$$

where $\mathbf{T}$ is the $(4 \times 4)$ frequency-dependent transfer matrix which is real in the absence of damping. It follows that the matrix $\mathbf{T}$ and, more specifically, its invariants summarize all the propagation features of the periodic cell.

\subsection{PROPAGATION REGIONS ON THE INVARIANTS' PLANE}

Let us consider a generic undamped bi-coupled periodic structure; due to the spectral properties of the symplectic matrix $\mathbf{T}[13,14]$ (see Appendix A), the characteristic equation $\operatorname{det}[\mathbf{T}-\lambda \mathbf{I}]=0$ reads as

$$
\lambda^{4}+I_{1} \lambda^{3}+I_{2} \lambda^{2}+I_{1} \lambda+1=0, \quad I_{1}, I_{2} \in \mathbb{R},
$$

where the coefficients $I_{1}$ and $I_{2}$ are the invariants of $\mathbf{T}$. Therefore, the reversibility property halves the number of the transfer matrix invariants.

The meaning of the eigenvalues $\lambda_{i}$ emerges from Floquet's theorem: there exist free wave motions (characteristic waves) in which $\mathbf{z}_{k+1}=\lambda_{i} \mathbf{z}_{k}$, each associated with an eigenvalue of T. If $\left|\lambda_{i}\right|<1$ the wave amplitude decays in the positive direction (forward wave), if $\left|\lambda_{i}\right|>1$ it decays in the negative direction (backward wave), if $\left|\lambda_{i}\right|=1$ no attenuation exists in the two directions. Due to the reversibility of the coefficients of equation (2), if $\lambda_{i}$ is an eigenvalue, then $\lambda_{i}{ }^{1}$ is also an eigenvalue (called the adjoint eigenvalue). Therefore forward and backward waves always exist in pairs and both have the same propagation properties. It follows that two eigenvalues $\lambda_{1}$ and $\lambda_{2}$ such that $\left|\lambda_{i}\right| \leqslant 1(i=1,2)$, completely define the propagation properties of a bi-coupled periodic structure; they will be here referred to as principal eigenvalues. Since the eigenvalues must be complex conjugates in pairs, if $\lambda_{1}$ is complex, then $\lambda_{2}=\bar{\lambda}_{1}$; if $\lambda_{1}$ is real, $\lambda_{2}$ is either real or complex and it has unit modulus. When a complex eigenvalue has unit modulus, its adjoint coincides with the complex conjugate, so that more than two eigenvalues satisfy the inequality $\left|\lambda_{i}\right| \leqslant 1$. To avoid indeterminacies, two eigenvalues with positive phase $0 \leqslant \vartheta \leqslant \pi$ will be taken as principal eigenvalues. If $\vartheta=0$ or $\pi$, only one of the two coincident eigenvalues must be taken. As usually done in the literature, previous findings can be restated in terms of the propagation constants $\mu_{i}$, instead of the eigenvalues $\lambda_{i}$, by defining $\lambda_{i}=\mathrm{e}^{\mu_{i}}$. This position maps the unit circle of the $\operatorname{Re}\left(\lambda_{i}\right)-\operatorname{Im}\left(\lambda_{i}\right)$ plane into the left half-space of the $\operatorname{Re}\left(\mu_{i}\right)-\operatorname{Im}\left(\mu_{i}\right)$ plane.

To solve (2) it is convenient to rewrite it in terms of the propagation constant $\mu$. By letting $\lambda=\mathrm{e}^{\mu}$ and multiplying by $\mathrm{e}^{2 \mu}$, equation (2) reads

$$
\mathrm{e}^{2 \mu}+I_{1} \mathrm{e}^{\mu}+I_{2}+I_{1} \mathrm{e}^{\mu}+\mathrm{e}^{2 \mu}=0,
$$

leading to the following quadratic equation in $\cosh \mu$ :

$$
4 \cosh ^{2} \mu+2 I_{1} \cosh \mu+I_{2}-2=0 .
$$

The roots of equation (4) are

$$
(\cosh \mu)_{1,2}=\frac{1}{4}\left(-I_{1} \pm \sqrt{8}+I_{1}^{2}-4 I_{2}\right)=: F_{1,2}\left(I_{1}, I_{2}\right) .
$$

To discuss equation (5) the cases $F_{1,2} \in \mathbb{R}$ and $F_{1,2} \in \mathbb{C}$ are separately considered. As far as $F_{1,2} \in \mathbb{R}$, if $F_{1,2} \in \mathscr{I}:=[-1,1]$, then $\mu=\mathrm{i} \vartheta$ and $\lambda=\mathrm{e}^{\mathrm{i} \vartheta}$ is complex with unit modulus; if $F_{1,2} \notin \mathscr{I}, \mu=\alpha+\mathrm{ij} \pi$ with $j$ integer, and $\lambda= \pm \mathrm{e}^{\alpha}$ is real. On the other hand, if $F_{1,2} \in \mathbb{C}$, $\mu=\alpha+\mathrm{i} \vartheta$ and $\lambda=\mathrm{e}^{\alpha+\mathrm{i} \vartheta}$ is complex with modulus different from 1 . The possible location of the eigenvalues on the complex plane are summarized in Figure 1. The wave 


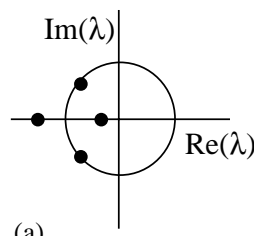

(a)

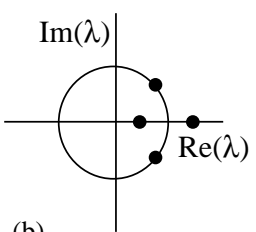

(b)

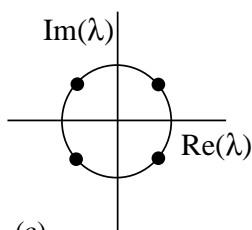

(c)

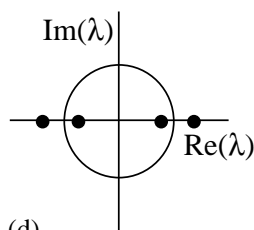

(d)

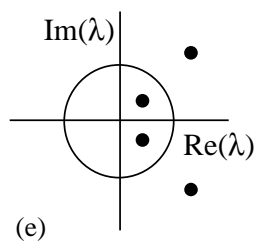

(e)

Figure 1. Allowable scenarios for $\lambda . \mathscr{I} \quad[1,1]$. (a) (d) $F_{1,2} \in \mathbb{R}$; (a) $F_{1} \notin \mathscr{I}, F_{2} \in \mathscr{I}$; (b) $F_{1} \in \mathscr{I}, F_{2} \notin \mathscr{I}$; (c) $F_{1,2} \in \mathscr{I} ;$ (d) $F_{1,2} \notin \mathscr{I} ;$ (e) $F_{1,2} \in \mathbb{C}$.

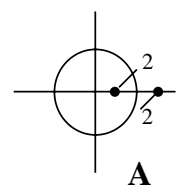

A

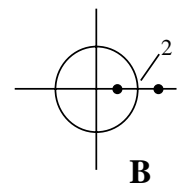

B

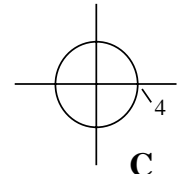

C

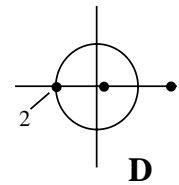

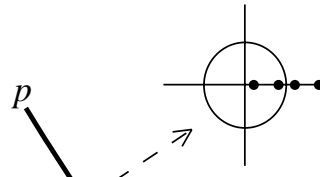
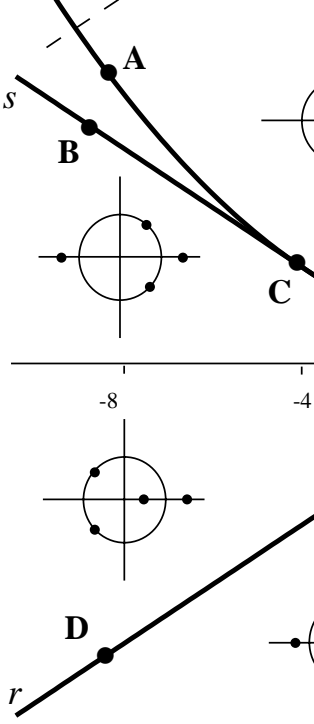

B
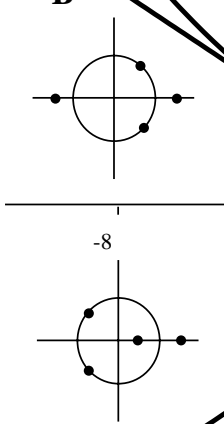
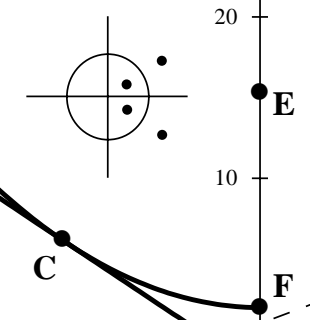

$\mathbf{F}$

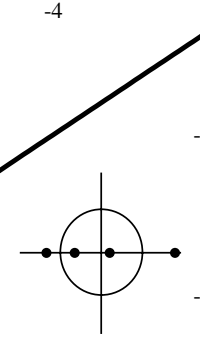

$\mathbf{G}$

$-10-$

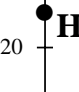

$\mathbf{H}$

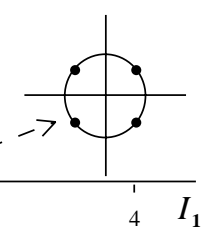

$-30$
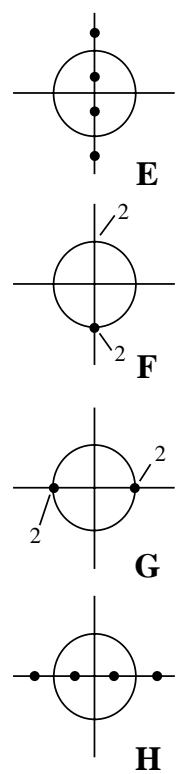

E

H

Figure 2. Position on the invariants' plane $I_{1} I_{2}$ according to the location of the eigenvalues $\lambda$ on the complex plane.

propagation characteristics of bi-coupled periodic systems can be conveniently described through a geometric representation on the invariants' plane $I_{1}-I_{2}$ (Figure 2). When $\cosh \mu=\mp 1$, two curves are obtained in the $I_{1}-I_{2}$ plane, given, respectively, by

$$
r:=\left\{\left(I_{1}, I_{2}\right) \mid 2-2 I_{1}+I_{2}=0\right\} ; s:=\left\{\left(I_{1}, I_{2}\right) \mid 2+2 I_{1}+I_{2}=0\right\} .
$$

A further curve, dividing the real roots of equation (5) from the complex ones, is given by the parabola

$$
p:=\left\{\left(I_{1}, I_{2}\right) \mid 8+I_{1}^{2}-4 I_{2}=0\right\} .
$$

The three curves $r, s$ and $p$ divide the invariants' plane into domains (propagation zones) where the eigenvalues are of the same type. In Figure 3 the propagation zones are labelled 


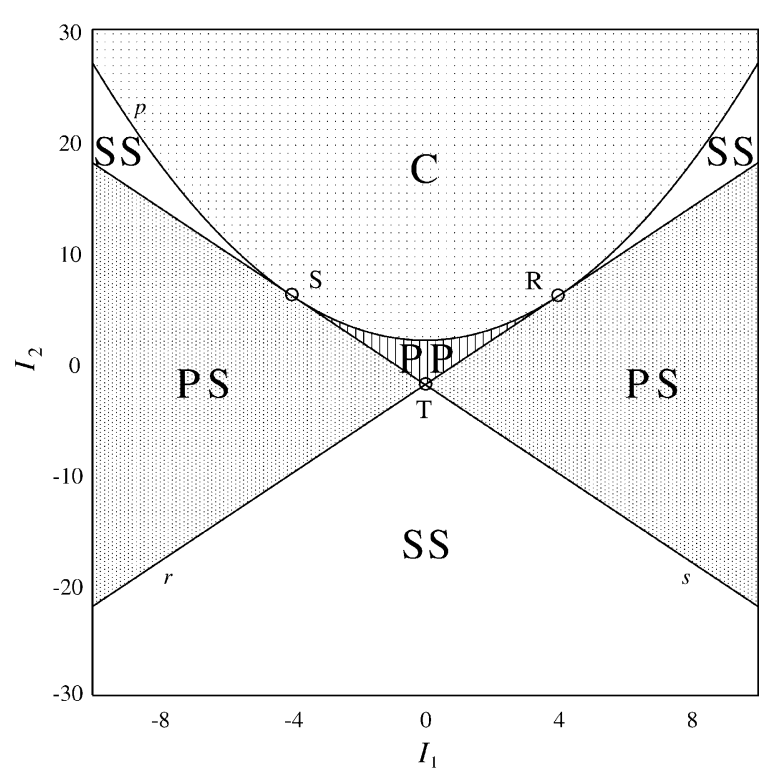

Figure 3. Propagation zones on the invariants' plane $I_{1} I_{2}$.

according to the notation commonly used in the literature. The region where both the pairs of $\lambda$ lie on the unit circle is referred to as pass-pass (PP); the regions where only one pair of $\lambda$ lies on the unit circle while the other pair is real are referred to as pass-stop (PS); the regions where only real pair of eigenvalues occurs are the stop-stop (SS) domains. Moreover, the curve $p$ bounds the so-called complex region (C) characterized by complex conjugate eigenvalues. It is worth noting that only the PP region is bounded while the remaining domains are unbounded.

In Appendix B the analysis so far addressed to bi-coupled structures is extended to the case of three-coupled periodic structures. The main steps of the analysis are sketched with the aim to highlight that the propagation properties of such systems can be entirely inferred by considering a three-dimensional invariants' space.

\subsection{ASYMPTOTIC BEHAVIOR OF THE ROOTS $\lambda$ ON THE INVARIANTS' PLANE}

In technical applications it is not rare that, as a physical parameter varies, the invariants $I_{1}$ and/or $I_{2}$ rapidly tend to infinity. Therefore, the analysis of the asymptotic behavior of the roots $\lambda$ of equation (2) as the invariants $I_{1}, I_{2}$ tend to infinity, either simultaneously or one at a time, is worth being investigated. In this section three limiting cases will be discussed, namely:

$$
\text { (a) }\left(I_{1}, I_{2}\right) \rightarrow \pm \infty, \quad(b) I_{1} \rightarrow \pm \infty, I_{2}=\mathcal{O}(1), \quad(c) I_{1}=\mathcal{O}(1), I_{2} \rightarrow \pm \infty \text {. }
$$

Case (a). By setting $I_{1}=\hat{I}_{1} / \varepsilon, I_{2}=\hat{I}_{2} / \varepsilon$, where $\varepsilon$ denotes a small quantity and $\mathcal{O}\left(\hat{I}_{1}\right)=\mathcal{O}\left(\hat{I}_{2}\right)=1$, substituting into equation (2), and dropping the hats, the equation reads as

$$
\lambda\left(I_{1} \lambda^{2}+I_{2} \lambda+I_{1}\right)+\varepsilon\left(\lambda^{4}+1\right)=0 .
$$


Two roots of $\mathcal{O}(1)$, one of $\mathcal{O}(\varepsilon)$ and one of $\mathcal{O}(1 / \varepsilon)$ are found (the latter by setting $\lambda=\varepsilon \hat{\lambda}$ and $\lambda=\hat{\lambda} / \varepsilon)$; they are, respectively,

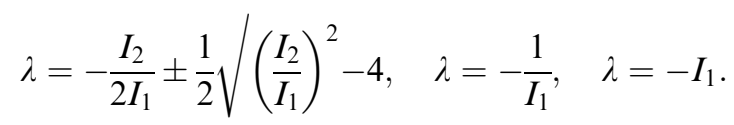

Case (b). By letting $I_{1}=\hat{I}_{1} / \varepsilon$ and substituting into equation (2) we get

$$
\varepsilon\left(\lambda^{4}+I_{2} \lambda^{2}+1\right)+I_{1}\left(\lambda^{3}+\lambda\right)=0
$$

Again, there exist two roots of $\mathcal{O}(1)$, one of $\mathcal{O}(\varepsilon)$ and one of $\mathcal{O}(1 / \varepsilon)$ and they are given by

$$
\lambda= \pm \mathrm{i}, \quad \lambda=-\frac{1}{I_{1}}, \quad \lambda=-I_{1} .
$$

The roots coincide with those of the previous case in which $I_{2}=0$.

Case (c). By letting $I_{2}=\hat{I}_{2} / \varepsilon^{2}$ and substituting into equation (2) it follows that

$$
\varepsilon^{2}\left(\lambda^{4}+I_{1} \lambda^{3}+I_{1} \lambda+1\right)+I_{2} \lambda^{2}=0 .
$$

The four roots of the latter equation, two of $\mathcal{O}(1 / \varepsilon)$ and two of $\mathcal{O}(\varepsilon)$ are given, respectively, by

$$
\lambda^{2}=-\frac{1}{I_{2}}, \quad \lambda^{2}=-I_{2}
$$

From the above results it follows that complex zones can exist only if $I_{2} \rightarrow+\infty$ (Case (c)) and they cannot exist whenever $I_{1} \rightarrow \pm \infty$ because at least one pair of eigenvalues is real (Cases (a) and (b)). Such conclusions are consistent with equation (7) based on which complex zones can only exist if $\left|I_{1}\right| \leqslant \sqrt{ }\left(4 I_{2}-8\right)$ while $I_{2} \geqslant 2$. The accuracy of the approximated formulas (9), (11) and (13) has been tested in Figure 4, where the real and the imaginary parts of the principal eigenvalues have been plotted along three straight paths on the invariant plane. The approximated solution is in very good agreement with exact (numerical) results as soon as the invariants are of $\mathcal{O}(10)$.

\subsection{ISO ATTENUATION CURVES ON THE INVARIANTS' PLANE}

As previously mentioned, the propagating or attenuating nature of the characteristic waves travelling through periodic systems is characterized by the modulus $\rho:=\left|\lambda_{i}\right|$ of the transfer matrix eigenvalues. Except for the PP zone, where both the waves propagate, attenuation of one or both the characteristic waves occurs on the whole invariant plane. In this section, attention is focused on describing the loci of the eigenvalues having the same modulus on the invariants' plane (iso-attenuation curves). From a geometrical point of view, the ensemble of these curves can be interpreted as the contour plot of the surface $\rho=\rho\left(I_{1}, I_{2}\right)$, shown in Figure 5(a).

By setting $\lambda=\rho \mathrm{e}^{\mathrm{i} \gamma}(0<\rho \leqslant 1,0 \leqslant \vartheta \leqslant \pi)$ and separating the real and imaginary parts in equation (2), the following two equations are obtained:

$$
\begin{gathered}
\left(\rho^{2} \cos 3 \vartheta+\cos \vartheta\right) I_{1}+\rho \cos 2 \vartheta I_{2}=-\frac{1}{\rho}-\rho^{3} \cos 4 \vartheta, \\
\left(\rho^{2} \sin 3 \vartheta+\sin \vartheta\right) I_{1}+\rho \sin 2 \vartheta I_{2}=-\rho^{3} \sin 4 \vartheta .
\end{gathered}
$$

By fixing $\rho$ and letting $\vartheta$ vary, equations $(14,15)$ yield the parametric equations $I_{1}=I_{1}(\vartheta)$, $I_{2}=I_{2}(\vartheta)$ of the iso- $\rho$ curves sought. They are depicted in Figure 5(b) while the details of the solution are given in Appendix C. Inside the complex region $\mathrm{C}$, each point of the curves $\rho=$ const is associated with a distinct value of $\vartheta \neq(0, \pi)$. When $\vartheta=(0, \pi)$, equations $(14,15)$ admit infinite solutions represented by the two families of straight lines with 


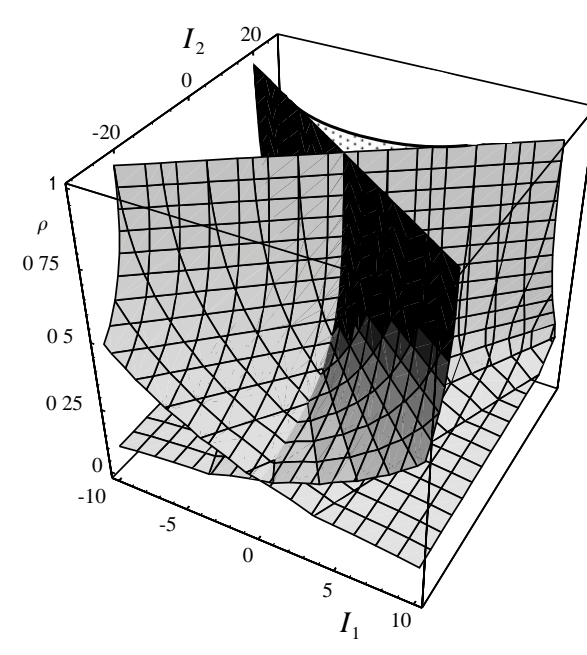

(a)

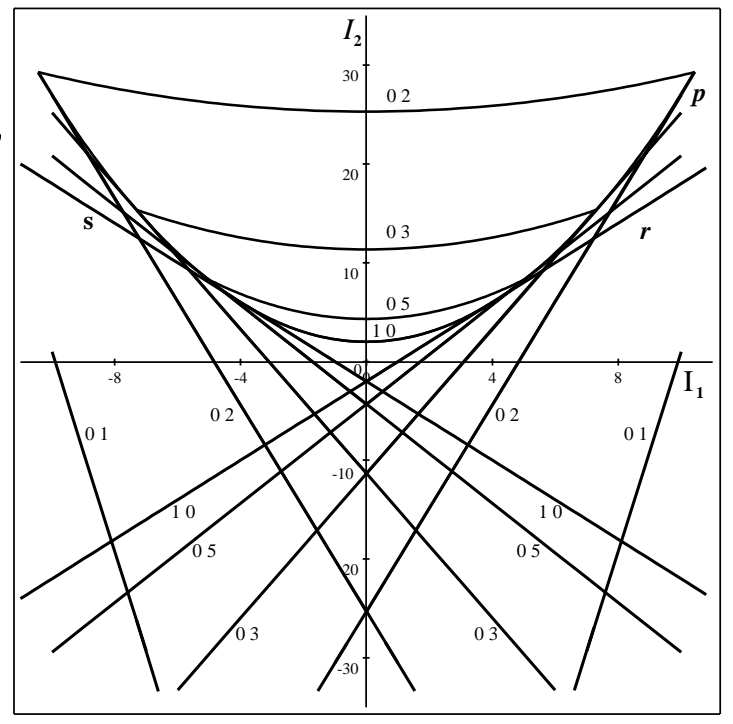

(b)

Figure 5. Modulus $\rho$ of the eigenvalues of T: (a) surface $\rho \quad \rho\left(I_{1}, I_{2}\right)$; (b) iso attenuation curves on the invariants' plane $I_{1} I_{2}$.

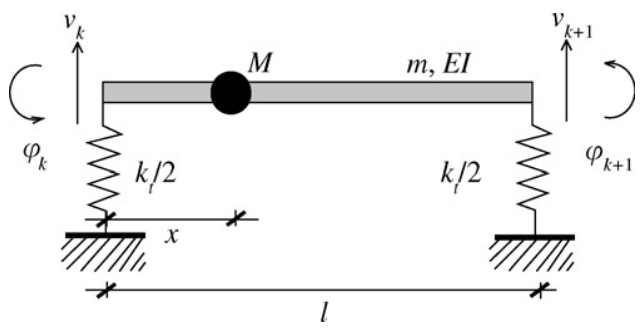

Figure 6. Bi coupled beam element.

a specific problem, two steps must be performed after deriving the transfer matrix T; first, the invariants must be expressed in terms of the physical parameters; then, the propagation regions (and possibly the iso-attenuation curves) must be mapped in a plane of two control parameters suitably selected. The procedure is illustrated here referring to a specific class of bi-coupled periodic structures whose repetitive elements, as sketched in Figure 6, are given by Euler beams of length $l$, flexural stiffness $E I$, resting on elastic supports with translational stiffness $k_{t} / 2$, with distributed mass $m$ and a lumped mass $M$ located at $x$. For such periodic elements, the state vector at the coupling point $k$ is given by $\mathbf{z}_{\mathbf{k}}=\left(v_{k}, \varphi_{k}, V_{k}, M_{k}\right)^{\mathrm{T}}$, where $v, \varphi$ and $V, M$ represent the generalized displacement and forces components respectively. The following four non-dimensional parameters govern the propagation properties of a cell:

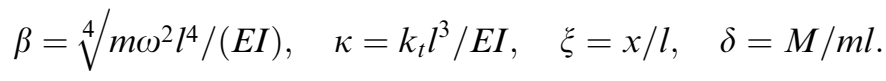

Different sub-models will be considered, namely: no lumped mass (two-parameter $\beta, \kappa$ model); mass located at mid-span (three-parameter $\beta, \kappa, \delta$ model); free mass location (four-parameter $\beta, \kappa, \xi, \delta$ model). The derivation of the transfer matrix for the fourparameter model is summarized in Appendix D. 


\subsection{TWO PARAMETER MODEL}

The first class of beams considered in this section refers to systems with distributed mass only. The model physical parameters are $\beta$ and $\kappa$. From the expression of the transfer matrix (see e.g. reference [15]), the following invariants are drawn:

$$
\begin{aligned}
& I_{1}=-2 \cosh \beta-2 \cos \beta-\frac{\kappa}{2 \beta^{3}}(\sin \beta-\sinh \beta), \\
& I_{2}=2+4 \cos \beta \cosh \beta+\frac{\kappa}{\beta^{3}}(\cosh \beta \sin \beta-\cos \beta \sinh \beta) .
\end{aligned}
$$

Equations (17) represent a non-linear mapping from points on the invariant plane $I_{1}-I_{2}$ to points on the physical parameters plane $\beta-\kappa$. Thus, the curves $r, s$ and $p$ of Figure 3 become the branches $r_{i}, s_{i}$ and $p_{i}$ of Figure 7, obtained by substituting (17) into equations $(6,7)$; in particular, parabola (7) is mapped into the closed curves $p_{i}$. Such branches define the propagation regions of the periodic structure. In Figure 7 closer views of the first three pass-pass zones are also presented. The points $T, S$ and $R$ of Figure 3 are mapped into the points $T_{i}, S_{i}$ and $R_{i}$, the index $i$ representing the $i$ th pass-pass region. The curves $r_{i}$ and $s_{i}$ are given by

$$
r_{i}, s_{i}:=\left\{(\beta, \kappa) \mid\left\{\begin{array}{ll}
1 \pm \cos \beta=0 & \text { for } i \text { odd } \\
\kappa=\frac{\mp 4 \beta^{3}(1 \pm \cosh \beta)}{\frac{\sin \beta}{(1 \pm \cos \beta)}(1 \pm \cosh \beta)-\sinh \beta} & \text { for i even }
\end{array}\right\}\right.
$$
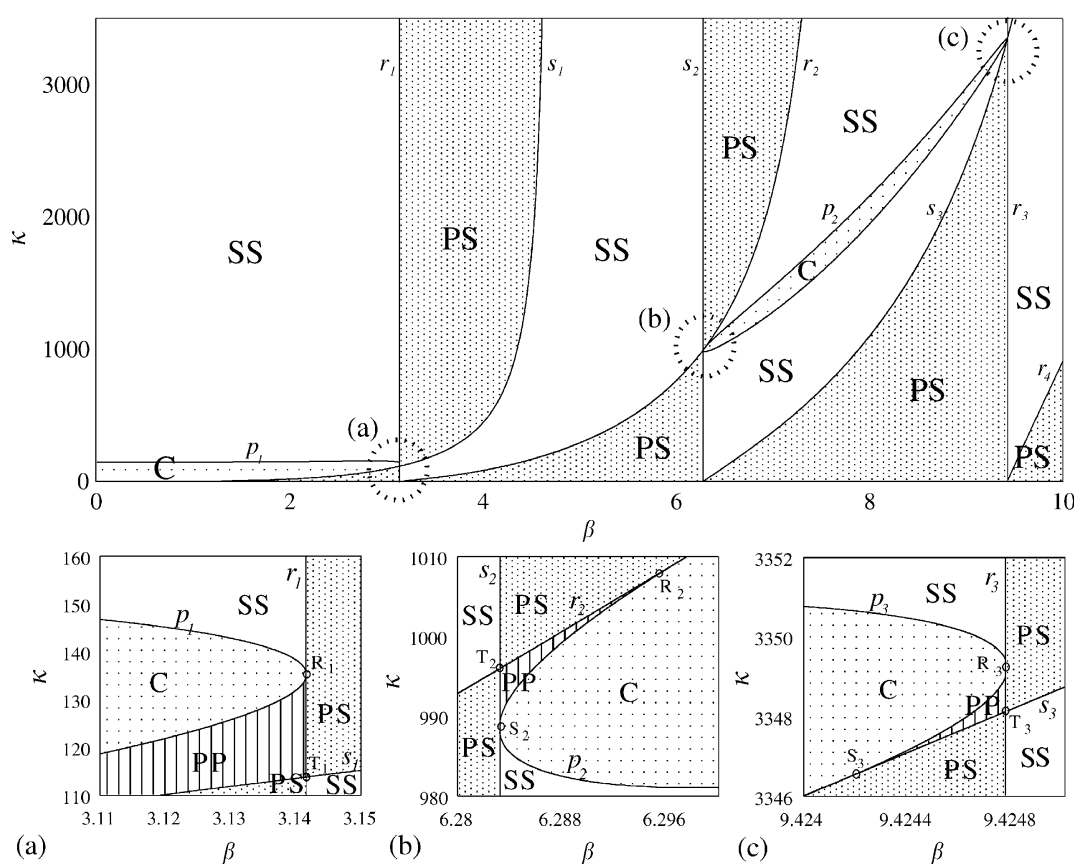

(b)

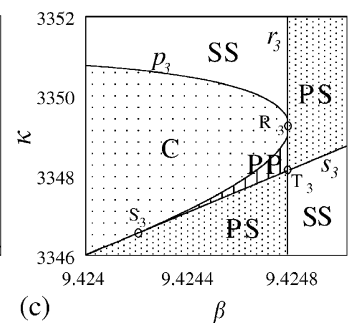

Figure 7. Propagation zones and closer views around the PP zones on the $\beta \kappa$ plane for the two parameter model. 
while the curves $p_{i}$ are made up of two branches, $p_{i u}$ (upper) and $p_{i l}$ (lower), defined in the intervals $\beta \in[n \pi,(n+1) \pi]$ ( $n$ even), having equations

$$
p_{i u, l}:=\left\{(\beta, \kappa) \mid \kappa=\frac{4 \beta^{3}(\cosh \beta-\cos \beta)}{(\sqrt{ } \sin \beta \pm \sqrt{ } \sinh \beta)^{2}}\right\} .
$$

As shown in Figure 8, the two branches $p_{i u}$ and $p_{i l}$ in equation (18) coincide for $\beta=n \pi$ and they tend to coalesce for large values of $\beta$; indeed, for $\beta \rightarrow \infty, p_{i u} \approx p_{i l} \approx 4 \beta^{3} / \tanh \beta \approx$ $4 \beta^{3}$. Such monotonically increasing trend implies that, for each value of the springs translational stiffness, only one complex region can be crossed. Furthermore, it can be inferred that, for this class of periodic structures, complex regions disappear as the frequency increases. Such result is consistent with the asymptotic analysis of the preceding section. Indeed, from the expression of $I_{1}$ in equation (17), it follows that as $\beta \rightarrow \infty$, $I_{1} \rightarrow-\infty$ so that at least one pair of real roots $\lambda$ will always exist (Cases (a) and (b) in section 2.2), preventing the eigenvalues from complex zones crossings.

The propagation zones shown in Figure 7 provide a complete description of the dependence of the wave propagation characteristics on the spring translational stiffness. The usual representation [4] of the real and imaginary parts of the propagation constants (Figures 9(a) and 9(b)) can be interpreted as section of the three-dimensional graphs of functions $\operatorname{Re} \mu=f(\beta, \kappa)$ and $\operatorname{Im} \mu=g(\beta, \kappa)$, obtained with a plane parallel to the $\beta$-axis at the chosen value of $\kappa$, whose trace is illustrated in Figure 9(c) for two values of $\kappa$; these traces map in the curves of Figure 9(d) on the invariants' plane. Thus, the non-linear mapping between the two planes allows one to represent the evolution of the arrangement of the propagation bands. In Figure 10 the iso-attenuation curves on the $\beta-\kappa$ plane are shown around the second PP zone (see Figure 7(b)). They have been obtained by using equations (17) in the solution of equations $(14,15)$ and solving for $\beta$ and $\kappa$. The original symmetry of Figure 5(b) is destroyed, whereas the strong gradient of the attenuation around the PP zone persists.

Finally, several mechanical considerations can be associated with either the curves $r$ and $s$ on the invariants' plane or, equivalently, with their representation $r_{i}, s_{i}$ on the physical parameters' plane. In 1975, Mead [4] presented the main results pertaining to multi-coupled periodic systems. Besides highlighting the existence of the complex bands, he identified the natural frequencies of a single symmetric element of a bi-coupled system with the bounding frequencies of the propagation bands on the $\beta$-axis. Since this result holds for any $\kappa$, it follows that along the line $r_{i}\left(s_{i}\right)$ are located both the natural frequencies corresponding to the symmetric modes of the single element when its translational
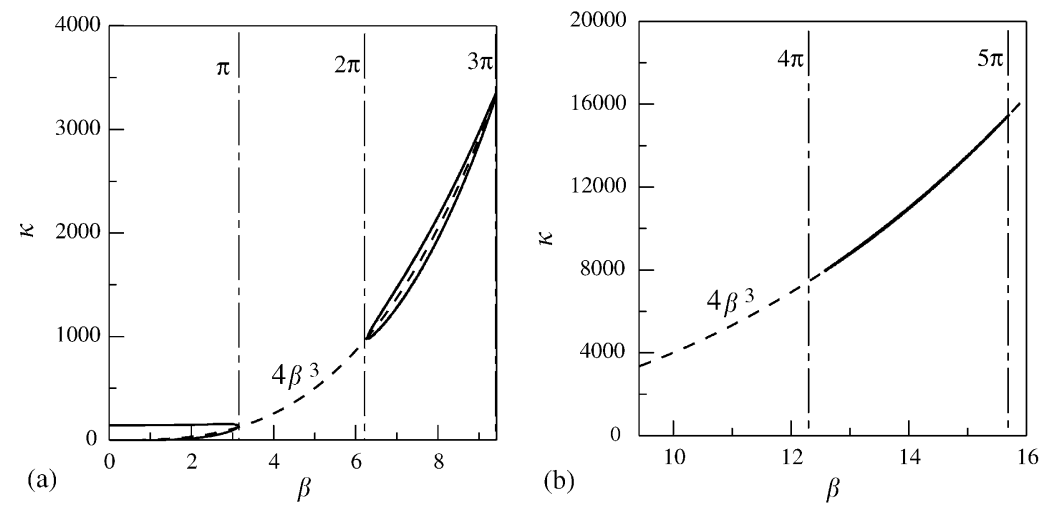

Figure 8. Complex zones on the $\beta \kappa$ plane: (a) $0 \leqslant \beta \leqslant 9.42$; (b) $9.42 \leqslant \beta \leqslant 16$. 


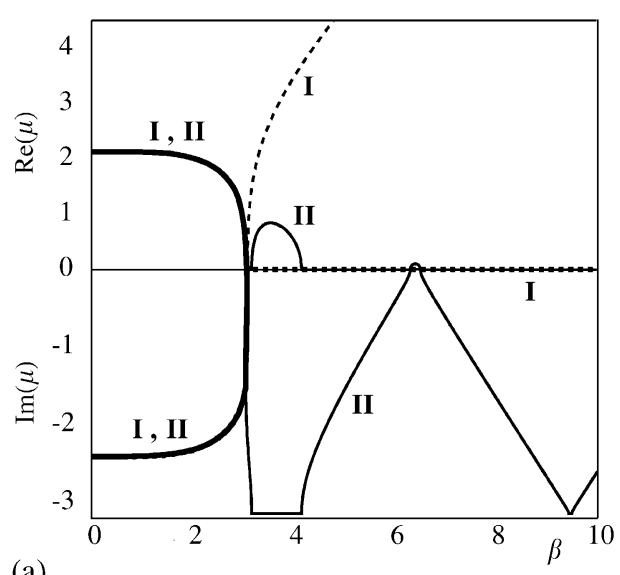

(a)

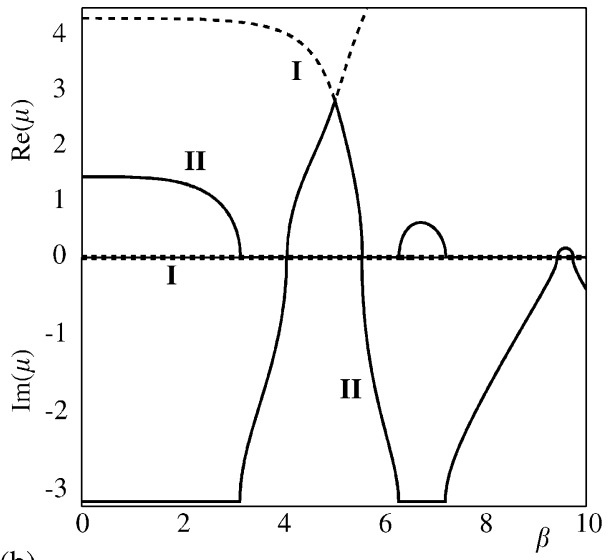

(b)
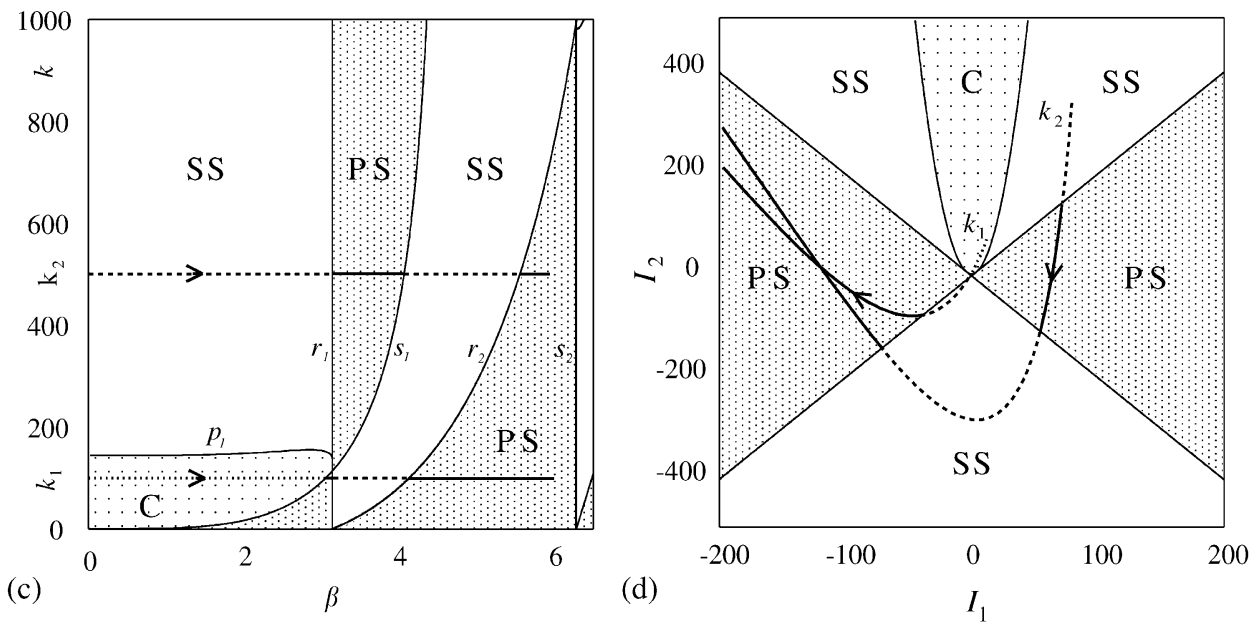

Figure 9. Real and imaginary parts of the propagation constants: (a) $\kappa \quad 100$; (b) $\kappa \quad 500$; and corresponding paths $\kappa$ const for increasing $\beta$; (c) on the physical plane; (d) on the invariants' plane.

(rotational) co-ordinates are locked and the natural frequencies corresponding to the antisymmetric modes of the single element when its rotational (translational) co-ordinates are locked (see Figure 11). Figure 11 explains the role of the parameter $\kappa$ in the sequence of the natural frequencies of the hinged-hinged and sliding-sliding beams. As expected, the parameter $\kappa$ does not affect the natural frequencies of the former.

\subsection{THREE PARAMETER MODEL}

Beams with a mid-span lumped mass are next considered, for which the propagation zones of Figure 12 are found for different mass ratios $\delta$. Comparison with Figure 7, representing the case $\delta=0$ highlights the effect of the parameter $\delta$. It can be noticed that, due to the mid-span location of the lumped mass, the value of $\delta$ does not affect the domain boundaries corresponding to the natural frequencies of the antisymmetric modes when either rotational or translational coordinates are locked. Furthermore, a contraction of the complex zones as $\delta$ increases can be noticed. For $\kappa=0$ and $\delta \neq 0$ propagation zones of the SS type exist. On the other hand, for $\kappa=0$ and $\delta=0$ such SS zones disappear; thereby we 


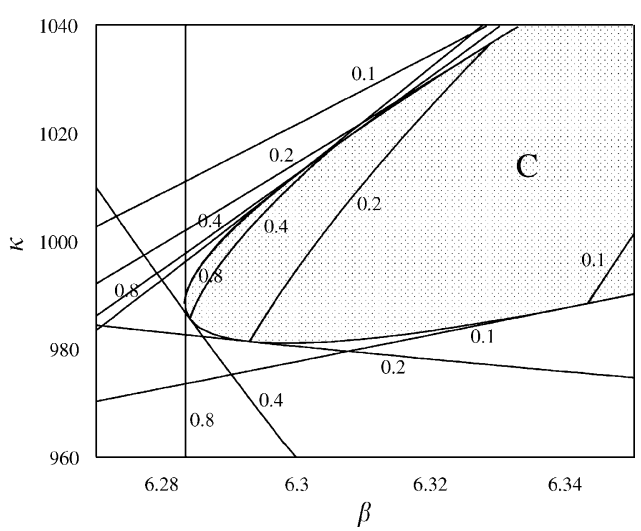

Figure 10. Iso attenuation curves on the $\beta \kappa$ plane.

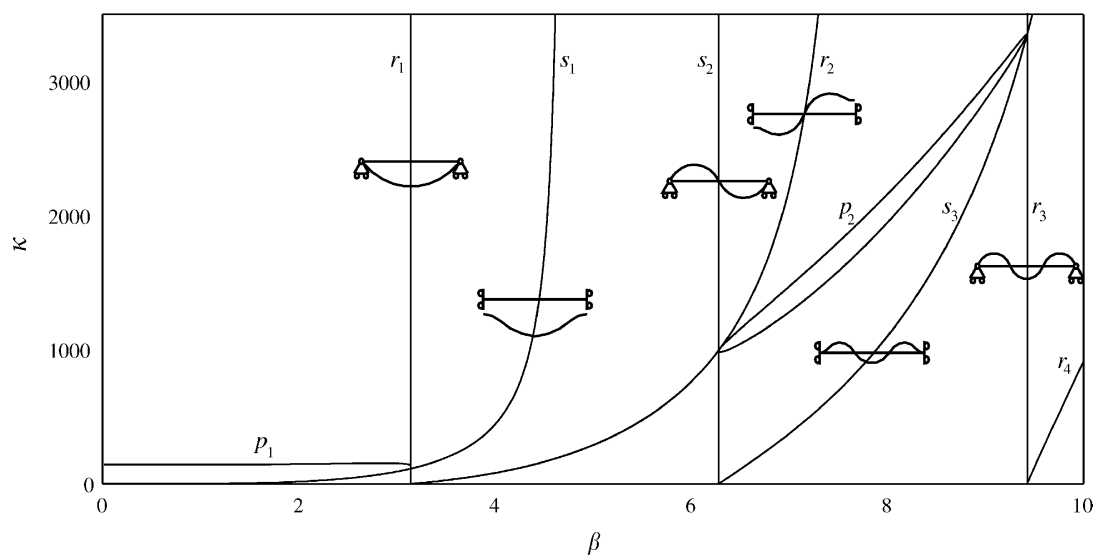

Figure 11. Bounding curves on the $\beta \kappa$ plane and natural frequencies of single elements.

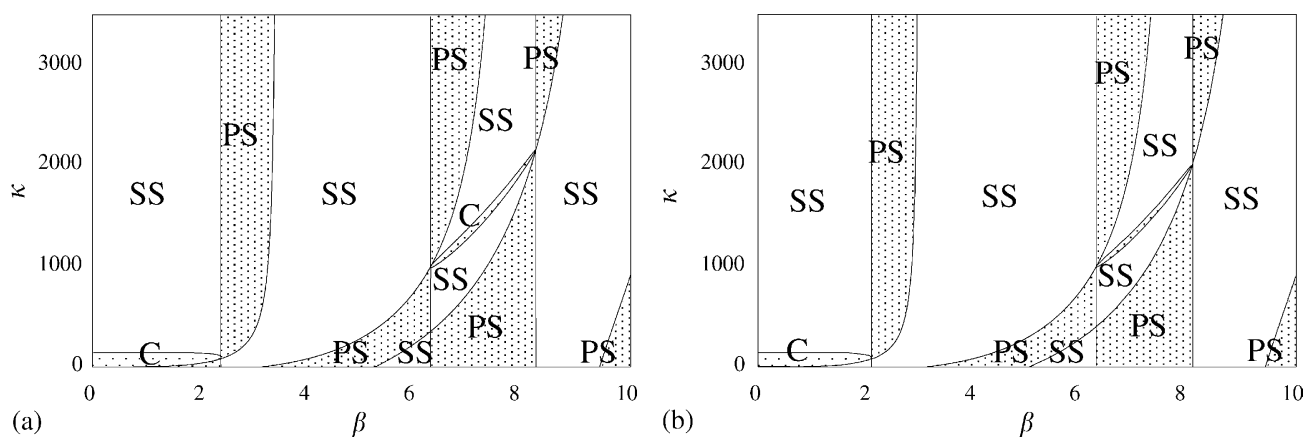

Figure 12. Propagation zones on the $\beta \kappa$ plane for the three parameter model: (a) $\delta \quad 1$; (b) $\delta 2$.

are left with a system whose period is arbitrary or, in other words, with a system behaving as being uniformly continuous where only PS zones can exist.

It might be of interest to investigate the representation of the propagation domains provided by a different choice of the control parameters. Figure 13 shows the propagation 


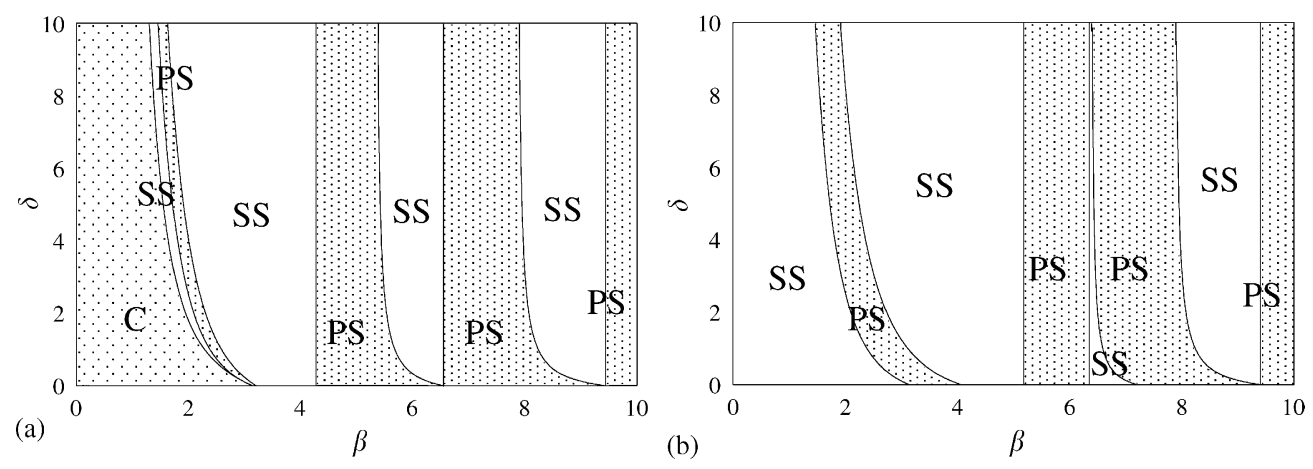

Figure 13. Propagation zones on the $\beta \delta$ plane for the three parameter model: (a) $\kappa \quad 125 ;$ (b) $\kappa \quad 500$.

domains in the $\beta-\delta$ plane for two values of the springs stiffness $\kappa$. As can be readily noticed, the propagation properties undergo qualitative variations depending on the considered stiffness value; for instance, the complex region found for $\kappa=125$ (Figure 13(a)) disappears for $\kappa=500$ (Figure 13(b)). Therefore, it can be concluded that the latter choice of physical parameters prevents a comprehensive qualitative description of the dynamic behavior of such class of bi-coupled periodic elements.

The circumstance outlined in section 2.2 pertaining to the tendency to infinity of the invariants is now shown for a simplified model. By assuming that the distributed mass of the beam vanishes $(m=0)$, the invariants $I_{1}, I_{2}$ assume the following manageable dimensional expression:

$$
\begin{aligned}
I_{1} & =\frac{1}{1152}\left(-4608+\frac{k_{t} l^{6}}{(E I)^{2}} M \omega^{2}-192 \frac{l^{3}}{E I} M \omega^{2}+384 \frac{k_{t} l^{3}}{E I}\right), \\
I_{2} & =-\frac{1}{576}\left(-3456+7 \frac{k_{t} l^{6}}{(E I)^{2}} M \omega^{2}+384 \frac{l^{3}}{E I} M \omega^{2}-768 \frac{k_{t} l^{3}}{E I}\right) .
\end{aligned}
$$

It follows that for such elements complex zones can exist only for $k_{t}$ and either $\omega$ or $M$ small; indeed, as one of the above-mentioned parameters increases, then $I_{1} \rightarrow \infty$. The above result holds also by assuming $l$ small; in the limit $l \rightarrow 0$, the system behaves as a beam resting on a spring bed whose characteristic exponents are complex conjugate.

\subsection{FOUR PARAMETER MODEL}

The bi-coupled asymmetric periodic structure considered in this section is built up by the repetitive elements shown in Figure 6, with a lumped mass $M$ located at an arbitrary position, so that its invariants depend on four parameters, $I_{i}=I_{i}(\beta, \kappa, \delta, \xi)$. The propagation regions on the control parameter plane $\beta-\kappa$ are shown in Figure 14 for different positions $\xi$ of the mass and a fixed mass ratio $\delta=1$. For $\xi=\frac{1}{2}$, the propagation zones coincide with those in Figure 12(a). The qualitative arrangement of the propagation domains can still be thoroughly described on this plane. In particular, for $\kappa=0$ the sequence of PS and SS bands, as $\beta$ increases, does not depend on $\xi$ because, once the spring supports have been removed, the system spatial periodicity is only governed by the relative distance between the masses. It is also worth mentioning that, for unsymmetric elements $\left(\xi \neq \frac{1}{2}\right)$, the natural frequencies can no longer be identified with the boundaries of the propagation zones. 

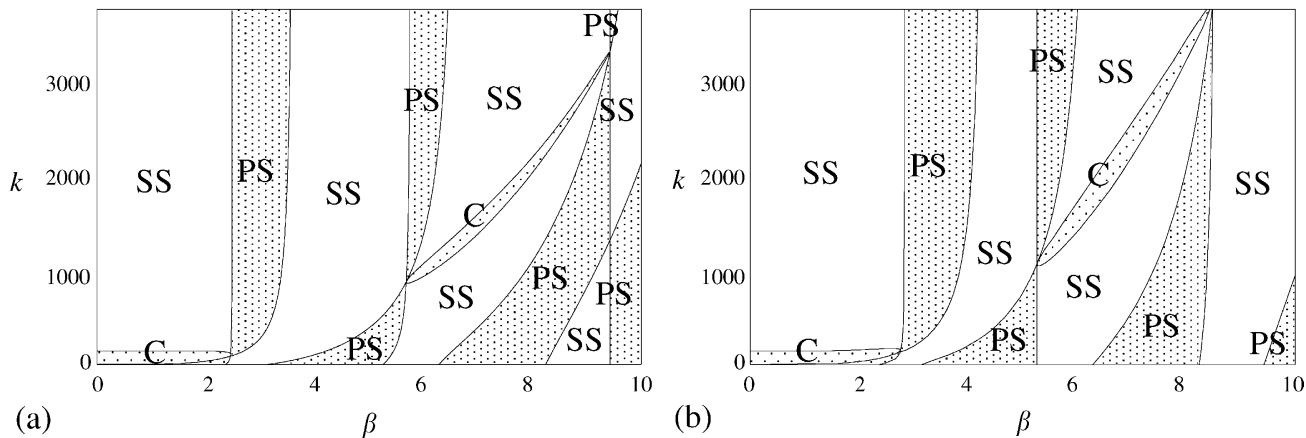

(b)

$\beta$

Figure 14. Propagation zones on the $\beta \kappa$ plane for the four parameter model $\left(\begin{array}{lll}\delta & 1) \text { : (a) } \xi & 0.33 \text {; (b) } \xi\end{array}\right.$ 0.16 .
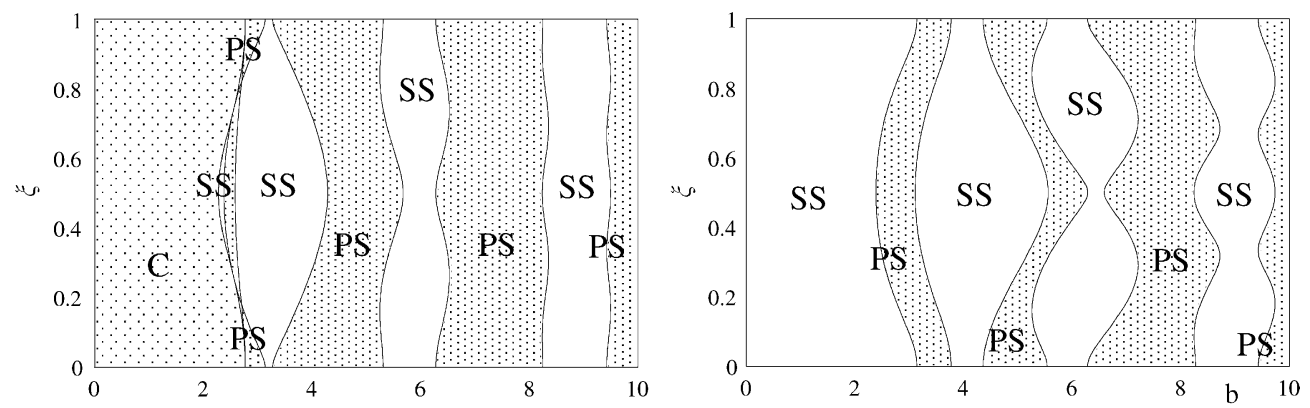

(a)

(b)
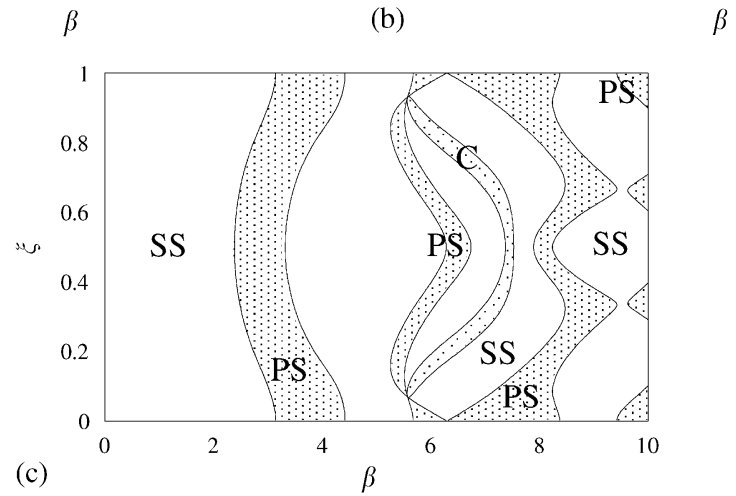

Figure 15. Propagation zones on the $\beta \xi$ plane for the four parameter model $(\delta \quad 1)$ : (a) $\kappa \quad 125 ;$ (b) $\kappa \quad 500$; (c) $\kappa 1600$.

In the four-parameter beam model the position $\xi$ of the lumped mass (or the mass ratio $\delta$ ) could be used as an alternative control parameter to describe the propagation domains. Figure 15 shows the domains on the $\beta-\xi$ plane for different $\kappa$ 's. Further representations on the $\kappa-\xi$ plane are also shown in Figure 16 for different $\beta$ 's. It turns out that such representations are not appropriate for describing all the propagation properties of the system. Therefore, again, $\kappa$ and $\beta$ mainly govern its dynamic behavior.

\section{CONCLUSIONS}

The propagation properties of periodic structures are thoroughly described in spaces having the minimum dimension necessary to qualitatively characterize the type of 

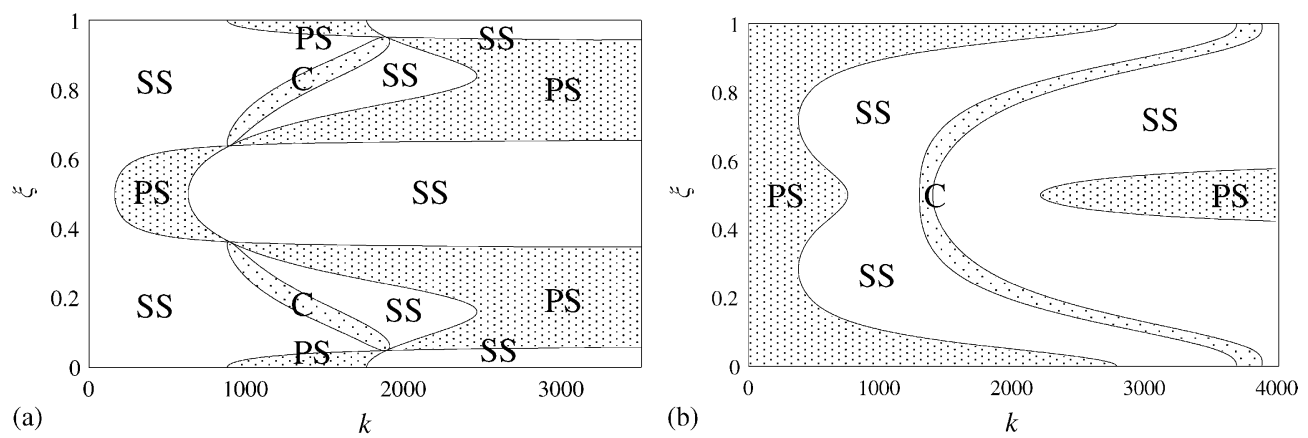

Figure 16. Propagation zones on the $\kappa \xi$ plane for the four parameter model $\left(\begin{array}{llll}\delta & 1\end{array}\right)$ (a) $\beta \quad 5.8 ;($ b) $\beta \quad 7$.

eigenvalues. Thus, moving from the characteristic equation of the symplectic transfer matrix, bi-coupled periodic structures have been analyzed on the invariants' plane. Such approach enabled one to derive analytically the boundaries of the stop, pass and complex domains. It has been found that pass-pass bands are confined to a small neighborhood of the origin. Furthermore, the values taken by the modulus of the eigenvalues (or, equivalently, by the real part of the propagation constants) have been portrayed on the same plane, showing that most of the characteristic waves attenuation occurs in the vicinity of the pass-pass zone boundaries. Then, periodic structures made up of beams resting on elastic supports have been considered. Various models characterized by the presence of different physical parameters have been studied. The free-wave propagation domains on different physical parameter planes have been obtained through non-linear mappings from the invariants' plane. Such representations highlighted the role played by each physical parameter on the propagation properties of the periodic structure.

\section{REFERENCES}

1. G. Sen Gupta 1970 Journal of Sound and Vibration 13, 89 101. Natural flexural waves and the normal modes of periodically supported beams and plates.

2. M. G. Faulkner and D. P. Hong 1985 Journal of Sound and Vibration 99, 29 42. Free vibrations of mono coupled periodic system.

3. D. J. MeAd 1975 Journal of Sound and Vibration 40, 1 18. Wave propagation and natural modes in periodic systems: I mono coupled systems.

4. D. J. Mead 1975 Journal of Sound and Vibration 40, 19 39. Wave propagation and natural modes in periodic systems: II bi coupled systems, with and without damping.

5. J. Signorelli and A. H. von Flotow 1988 Journal of Sound and Vibration 126, 127 144. Wave propagation, power flow, and resonance in a truss beam.

6. Y. YONG and Y. K. LiN 1989 Journal of Sound and Vibration 129, 99 118. Propagation of decaying waves in periodic and piecewise periodic structures of finite length.

7. D. J. MeAd 1970 Journal of Sound and Vibration 11, 181 197. Free wave propagation in periodically supported infinite beams.

8. D. Bouzit and C. Pierre 2000 Chaos, Solitons and Fractals 11, 1575 1596. Wave localization and conversion phenomena in multi coupled multi span beams.

9. D. J. MeAD 1986 Journal of Sound and Vibration 104, 9 27. A new method of analyzing wave propagation in periodic structures; applications to periodic Timoshenko beams and stiffened plates.

10. A. S. Bansal 1997 Journal of Sound and Vibration 207, 365 382. Free waves in periodically disordered systems: natural and bounding frequencies of unsymmetric systems and normal mode localization.

11. G. H. Koo and Y. S. PARK 1998 Journal of Sound and Vibration 210, 53 68. Vibration reduction by using periodic supports in a piping system. 
12. J. Guckenheimer and P. J. Holmes 1983 Nonlinear Oscillations, Dynamical Systems and Bifurcations of Vector Fields. New York: Springer Verlag.

13. W. X. Zhong and F. W. Williams 1992 Proceedings of the Institution of Mechanical Engineers, Part C 206, 371 379. Wave problems for repetitive structures and symplectic mathematics.

14. W. X. Zhong and F. W. Williams 1993 Computers and Structures 49, 749 750. Physical interpretation of the symplectic orthogonality of the eigensolutions of a Hamiltonian or symplectic matrix.

15. E. C. Pestel and F. A. Leckie 1963 Matrix Methods in Elastomechanics. New York: McGraw Hill.

\section{APPENDIX A: EIGENVALUES RECIPROCITY VIA DYNAMIC STIFFNESS MATRIX}

The eigenvalues reciprocity property of the transfer matrix of periodic structures can be derived by resorting to the dynamic stiffness matrix. According to this approach, the equation of motion of an undamped generic element reads as

$$
\left(\begin{array}{ll}
\mathbf{M}_{L L} & \mathbf{M}_{L R} \\
\mathbf{M}_{R L} & \mathbf{M}_{R R}
\end{array}\right)\left(\begin{array}{c}
\ddot{\mathbf{q}}_{L} \\
\ddot{\mathbf{q}}_{R}
\end{array}\right)_{k}+\left(\begin{array}{ll}
\mathbf{K}_{L L} & \mathbf{K}_{L R} \\
\mathbf{K}_{R L} & \mathbf{K}_{R R}
\end{array}\right)\left(\begin{array}{l}
\mathbf{q}_{L} \\
\mathbf{q}_{R}
\end{array}\right)_{k}=\left(\begin{array}{c}
\mathbf{f}_{L} \\
\mathbf{f}_{R}
\end{array}\right)_{k} .
$$

In the latter equation $\mathbf{q}_{i}$ and $\mathbf{f}_{i}$, with $i=L, R$, denote the $n$-dimensional displacement and external forces vectors respectively. The suffixes $L$ and $R$ indicate the left and right ends, respectively, of the repetitive element and the terms $\mathbf{M}_{i j}, \mathbf{K}_{i j}(i, j=L, R)$ represent the $(n \times n)$ mass and stiffness submatrices respectively. Index $k$ refers to the $k$ th element, joining joints $k$ and $(k+1)$. By denoting with $\mathbf{q}_{k}$ the displacements at the joint $k$ and enforcing the compatibility between the adjacent elements, $\mathbf{q}_{L, k}=\mathbf{q}_{R, k 1}=\mathbf{q}_{k}$ and $\mathbf{q}_{R, k}=$ $\mathbf{q}_{L, k+1}=\mathbf{q}_{k+1}$ results; by taking into account the symmetry of mass and stiffness matrices, the equations of motion of the periodic structure are

$$
\mathbf{B} \ddot{\mathbf{q}}_{k 1}+\mathbf{A} \ddot{\mathbf{q}}_{k}+\mathbf{B}^{\mathrm{T}} \ddot{\mathbf{q}}_{k+1}+\mathbf{D} \mathbf{q}_{k 1}+\mathbf{C} \mathbf{q}_{k}+\mathbf{D}^{\mathrm{T}} \mathbf{q}_{k+1}=\mathbf{f}_{k},
$$

where $\mathbf{B}=\mathbf{M}_{R L}, \mathbf{D}=\mathbf{K}_{R L}, \mathbf{A}=\mathbf{M}_{L L}+\mathbf{M}_{R R}$ and $\mathbf{C}=\mathbf{K}_{L L}+\mathbf{K}_{R R}$. For harmonic motions $\mathbf{q}_{k}=\mathbf{X}_{k} \mathrm{e}^{\mathrm{i} \omega t} ;$ by defining $\mathscr{E}_{i} \mathbf{q}_{k} \doteq \mathbf{q}_{k+i}$, the following difference equation is obtained:

$$
\left[\left(\mathbf{D}-\omega^{2} \mathbf{B}\right) \mathscr{E}_{1}+\left(\mathbf{C}-\omega^{2} \mathbf{A}\right)+\left(\mathbf{D}-\omega^{2} \mathbf{B}\right)^{\mathrm{T}} \mathscr{E}_{1}\right] \mathbf{X}_{k}=\mathbf{0} .
$$

Equation (A.3) admits the solution $\mathbf{X}_{k}=\lambda^{k} \mathbf{u}$, leading to the following eigenvalue problem

$$
\left[\left(\mathbf{D}-\omega^{2} \mathbf{B}\right) \frac{1}{\lambda}+\left(\mathbf{C}-\omega^{2} \mathbf{A}\right)+\left(\mathbf{D}-\omega^{2} \mathbf{B}\right)^{\mathrm{T}} \lambda\right] \mathbf{u}=\mathbf{0} .
$$

The latter problem admits non-trivial solution if and only if the determinant of the matrix inside the brackets is equal to zero, i.e.,

$$
\operatorname{det}\left\{\left(\mathbf{D}-\omega^{2} \mathbf{B}\right) \frac{1}{\lambda}+\left(\mathbf{C}-\omega^{2} \mathbf{A}\right)+\left(\mathbf{D}-\omega^{2} \mathbf{B}\right)^{\mathrm{T}} \lambda\right\}=0 .
$$

If the above determinant is equal to zero, the same holds for the determinant of the transpose matrix:

$$
\operatorname{det}\left\{\left(\mathbf{D}-\omega^{2} \mathbf{B}\right) \lambda+\left(\mathbf{C}-\omega^{2} \mathbf{A}\right)+\left(\mathbf{D}-\omega^{2} \mathbf{B}\right)^{\mathrm{T}} \frac{1}{\lambda}\right\}=0
$$

in which, due to the symmetry of the mass and stiffness matrices, $\left(\mathbf{C}-\omega^{2} \mathbf{A}\right)^{\mathrm{T}}=$ $\left(\mathbf{C}-\omega^{2} \mathbf{A}\right)$. It follows from equations (A.5) and (A.6) that if $\lambda$ is a solution of (A.5) then $1 / \lambda$ is also a solution. Therefore, when the characteristic equation (A.5) is expanded, its coefficients are symmetric. 
The analysis of the undamped bi-coupled systems carried out in section 2 can be in principle extended to the three-coupled case, although the analysis is not straightforward. The characteristic equation (2) is now substituted by

$$
\lambda^{6}+I_{5} \lambda^{5}+I_{4} \lambda^{4}+I_{3} \lambda^{3}+I_{2} \lambda^{2}+I_{1} \lambda+1=0 .
$$

By setting $\lambda=\mathrm{e}^{\mu}, \mu \in \mathbb{C}$ and considering that $I_{4}=I_{2}$ and $I_{5}=I_{1}$, the latter expression becomes

$$
\left(\mathrm{e}^{3 \mu}+\mathrm{e}^{3 \mu}\right)+I_{1}\left(\mathrm{e}^{2 \mu}+\mathrm{e}^{2 \mu}\right)+I_{2}\left(\mathrm{e}^{\mu}+\mathrm{e}^{\mu}\right)+I_{3}=0,
$$

which is also written as

$$
2 \cosh 3 \mu+2 I_{1} \cosh 2 \mu+2 I_{2} \cosh \mu+I_{3}=0 .
$$

After some algebraic manipulation the latter equation can be expressed as a cubic equation in $\cosh \mu$ as follows:

$$
\cosh ^{3} \mu+\frac{1}{2} I_{1} \cosh ^{2} \mu+\frac{1}{4}\left(I_{2}-3\right) \cosh \mu+\frac{1}{8}\left(I_{3}-2 I_{1}\right)=0 .
$$

By changing the variable according to $\cosh \mu=\zeta-I_{1} / 6$, the original equation is transformed into the simpler form

$$
\zeta^{3}+p \zeta+q=0,
$$

where $p=-I_{1}^{2} / 3+I_{2}-3$ and $q=1 / 4\left(I_{3}-I_{1}\right)-1 / 6 I_{1} I_{2}-1 / 27 I_{1}^{3}$. For $p$ and $q$ real, the space $\left(I_{1}, I_{2}, I_{3}\right)$ is divided into three types of regions according to the sign of the discriminant $\Delta=q^{2} / 4+p^{3} / 27$ of equation (B.5), given by

$$
\Delta=\left[4 I_{1}^{3}+9 I_{1}\left(3+2 I_{2}\right)-27 I_{3}\right]^{2}-64\left(9+I_{1}^{2}-3 I_{2}\right)^{3} .
$$

For $\Delta>0$, there exist one real and two complex conjugate roots, for $\Delta<0$, there are three simple real roots, and for $\Delta=0$, there are a double and a simple real root.

\section{APPENDIX C: PARAMETRIC EQUATIONS OF THE ISO ATTENUATION CURVES}

The set of equations $(14,15)$ admits a unique solution for $0<\vartheta<\pi$ :

$$
\begin{gathered}
I_{1}(\rho, \vartheta)=-2(\rho+1 / \rho) \cos \vartheta, \\
I_{2}(\rho, \vartheta)=2 \cos 2 \vartheta+(\rho+1 / \rho)^{2} .
\end{gathered}
$$

Curves (C.1, C.2) lie inside the complex region C (see Figure 5(b)) and a distinct value of $\vartheta$ is associated with each point of such curves $\rho=$ const. On the other hand, for $\vartheta=(0, \pi)$, the set of equations $(14,15)$ provides infinite solutions consisting of the pairs $\left(I_{1}, I_{2}\right)$ such that

$$
\pm \rho\left(\rho^{2}+1\right) I_{1}+\rho^{2} I_{2}+\rho^{4}+1=0 .
$$

Curves (C.3) describe two families of straight lines, tangent to the parabola $p$, with negative and positive slopes respectively. Lines (C.3) belong to the PS and SS zones; in particular, both the families exist within the SS regions (two real eigenvalues) while only one family can exist within the PS region (one real eigenvalue). The same value $(0, \pi)$ of $\vartheta$ is associated with each point of such lines.

In order to show that the iso- $\rho$ curves encircle the PP zone, it suffices to verify that, when $\vartheta \rightarrow(0, \pi)$ the parametric curves (C.1,C.2) meet the straight lines (C.3) for the same 
$\rho$ value. Indeed, from (C.1,C.2),

$$
\lim _{\vartheta \rightarrow(0, \pi)} I_{1}=\mp 2(1 / \rho+\rho), \quad \lim _{\vartheta \rightarrow(0, \pi)} I_{2}=4+1 / \rho^{2}+\rho^{2},
$$

which satisfies equation (C.3). Since these values of $I_{1}$ and $I_{2}$ also satisfy equation (7), the intersection points belong to the parabola $p$.

\section{APPENDIX D: TRANSFER MATRIX FOR THE FOUR-PARAMETER MODEL}

The main steps pertaining to the derivation of the transfer matrix of the beam element sketched in Figure 6 are summarized in this section.

Due to the presence of the lumped mass, it is convenient to divide the integration domain of the differential problem $m \ddot{v}+E I v^{\prime \prime \prime \prime}=0$ into two intervals, to the left and to the right of the lumped mass located at the abscissa $x$. By letting $v_{i}=\phi_{i}(s) \mathrm{e}^{\mathrm{i} \omega t}(i=1,2)$, two space-dependent ordinary differential equations follow, namely $\phi_{i}^{\prime \prime \prime \prime}-a^{4} \phi_{i}=0$ $(i=1,2)$, where $a^{4}=m \omega^{2} / E I$. The solutions of such equations are expressed as

$$
\phi_{i}(s)=C_{i 1} \sin a s+C_{i 2} \cos a s+C_{i 3} \sinh a s+C_{i 4} \cosh a s, \quad i=1,2 .
$$

The eight constants $C_{i j}$ are found by solving an $(8 \times 8)$ algebraic problem obtained by imposing the nodal displacements and rotations at the left $\left(v_{L}, \varphi_{L}\right)$ and right $\left(v_{R}, \varphi_{R}\right)$ ends of the beam segment and enforcing the compatibility and equilibrium at the mass location $x$. Afterwards, the end forces are evaluated as functions of the end displacements through:

$$
\left\{\begin{array} { l } 
{ f _ { L } = E I \phi _ { 1 } ^ { \prime \prime \prime } ( 0 ) , } \\
{ m _ { L } = - E I \phi _ { 1 } ^ { \prime \prime } ( 0 ) , }
\end{array} \quad \left\{\begin{array}{l}
f_{R}=-E I \phi_{2}^{\prime \prime \prime}(l), \\
m_{R}=E I \phi_{2}^{\prime \prime}(l)
\end{array}\right.\right.
$$

from which the dynamic stiffness matrix $\mathbf{S}$ is derived. The elastic supports are taken into account by adding the spring translational stiffness to the diagonal terms $S_{i i}, i=1,3$. After partitioning the matrix $\mathbf{S}$ into $(2 \times 2)$ submatrices, the transfer matrix $\mathbf{T}$ is eventually obtained by using the well-known relations [13]: $\mathbf{T}_{L L}=-\mathbf{S}_{L R}^{-1} \mathbf{S}_{L L}, \mathbf{T}_{L R}=\mathbf{S}_{L R}^{-1}, \mathbf{T}_{R L}=$ $-\mathbf{S}_{R L}+\mathbf{S}_{R R} \mathbf{S}_{L R}^{-1} \mathbf{S}_{L L}, \mathbf{T}_{R R}=-\mathbf{S}_{R R} \mathbf{S}_{L R}^{-1}$. 